\title{
Image Texture Classification using Fuzzy Inclusion and Fuzzy Entropy Measures
}

\author{
M.Subba Rao ${ }^{1}$, Dr. B.Eswara Reddy ${ }^{2}$ \\ ${ }^{1}$ Department of Information Technology, Annamacharya Institute of Technology and Sciences, Rajampet, Kadapa, \\ A.P., INDAIA, msraoswap@gmail.com \\ ${ }^{2}$ Director, Software Development Centre, JNTUA, Anantapur, A.P., INDIA, eswarcsejntua@gmail.com
}

\begin{abstract}
Texture classification is one of the sort-out methods in pattern recognition. Selection of features plys a crucial role in enhancing Machine Learning efficiency as it significantly improves the performance of Texture classification by discarding insignificant features from the original set. Most of the Feature Selection techniques are statistical. They are not versatile to accommodate human thinking and thus the evolving demands and desires of real-life processes. We only make a choice between including and excluding a feature. In the very least, the fuzziness of human thought and perception is not known to enhance the collection of features and thus the precision of the classifier. Accuracy in database classification can be achieved through feature selection while at the same time can speed up the classification rate. The main objective of the work is to choose the most significant features in the feature set to perform given task. In this paper, Fuzzy Inclusion and Fuzzy Entropy measures are applied in feature extraction and the experimental results show that accuracy in classification is proved with other techniques. A comparison is formed between the prevailing methods and therefore the proposed method. The proposed method shows better results than existing methods with best classification rate.
\end{abstract}

Key words: Image Texture Classification, Feature Selection, Fuzzy Inclusion, Fuzzy Entropy, Accuracy

\section{INTRODUCTION}

Texture classification is the method by which specific textures are identified from the specified images. While the description of the textures themselves sometimes seems irrelevant in its own way, however, it is possible to implement a large number of real-life problems related to unique textures of various materials [1]. The texture is characterized by a nonuniform spatial distribution of image intensities. There are mainly three different ways of pattern recognition on Texture Classification [9]. Statistical, Structural and Neural Network Pattern Recognition Texture based classification methods are used in a number of applications in the real world, such as content-based image recovery [2], face recognition [3], rock classification, and wood species recognition, classification of fabrics and geographic segmentation of the landscape. The aim of the texture classification is to classify the sample image in one of a number of known texture classes. There are two types of texture classification that are supervised and unsupervised. In the supervised classification, the classifier is equipped with the characteristics of the known classes. In the unsupervised classification process, the classifier identifies various classes on the basis of the similarities of the input characteristics, so there is no previous preparation of the classifier.

Classification methods texture can be divided into three pixel-related categories based on local and local characteristics [4].Choosing a feature is the job of choosing the most suitable and typical features. The approach reduces several features by removing obsolete, irritating and least important features. Function collection is often made either globally or locally [5]. Global Feature Selection (GLF) methods measure the general importance of the feature regardless of its application to any individual class [6]. Local Feature Selection (LFS) methods are those that are determined separately on each eligible class and then the selection is based on distinct scores [6]. The selection strategies are often predictive and state the status of the feature as either essential or not essential. But in real situations, selections are not easy and take into account a variety of human uncertainties. This can happen due to a number of realities that could not be constrained by $[0,1]$ selection[7]. This outcome indicates that owing to this subjective existence of statistical techniques, the 
bulk of wrongly categorized data may also be accurately categorized. The literature further exposes the very fact that the majority of LFS approaches, which are local to individual classes, can be used to choose replicated features for different classes [8]. Additional tests will also be needed to resolve this problem, which and increases the computing costs. Then, the matching features collection with separate divisions can obstruct the output of the classifier.

The Image Texture Classification is applied in this Article with help of Fuzzy inclusion \&Fuzzy entropy measures. After translating the regional zone of a single image pixel $\mathrm{p}$ to a fuzzy set A, Researchers estimate its entropy \& unique enclosure values between a few typical fuzzy sets and the specified set A. This data or information returns every t threshold correlated with pixel p. It is not the best process but it's a fully integrated system and most useful and flexible process. According to inconsistent or poor illumination, the background light is not consistent within the document picture recorded by the camera, which is the only impracticable threshold-based process. In order to resolve the above shortcomings, a substitution approach using fuzzy sets is introduced by considering Fuzzy Inclusion and Fuzzy Entropy measures of fixed and variable membership values. Section 2 describes related research and its associated work and highlights its new aspects. Section 3 presents a more comprehensive proposed methodology to classify the images effectively. Results and Performance measurements are covered in Chapter $4 \&$ Conclusion in Chapter 5.

\section{LITERATURE SURVEY}

The academic group has experienced the exponential growth of interest in data mining (DM) in recent decades. Investigators concentrate primarily on various facets of DM, such as data abstraction, preprocessing, extraction of features, design, collection and classification of features. They use computational, empirical, heuristic, Meta heuristic and usual algorithms to impact the problems at hand. There are several experiments from the historical past that have focused on the collection of features for DM are identified. Number of experiments on the most widely utilized filter, wrapper and integrated Feature Selection (FS) methodologies has been conducted [8]. Microarray data is a genetic database that collects organic phenomena for different studies. The survey conducted includes a variety of FS approaches. The Particle Swarm Optimization (PSO) algorithm has been developed $[8,10]$.In each of these publications, the PSO was used for the collection of features for specific activities. The sentiment analysis has done based on the dimension derived from the various results. The PSO is used for the collection of features from the high dimensional real-life data source [10]. The approach suggested could be a trivial, computationally well-organized FS approach.

Feature Selection approach for dealing with the question of the imbalance class was introduced in [11]. The proposed solution, referred to as the improved global feature selection method, centered on addressing the issue of the mismatch class by choosing Same number of features in each group. Researchers were used to OR, but rather to assess the preference of each class, either negative or positive, and to choose the set of characteristics of each class. This suggested solution increased the appearance of the lowest form of FFS. The results of the report support the effectiveness of the novel technique. Subsequently, the shortcoming of the alternative strategy has been shown in [12].

Due to its rigidity, the solution previously proposed could disregard some extremely typical features of the popular class. This neglect would have an impact on instruction and, therefore, on the classifier's performance. A standardized vector function selection scheme which can be used to pick the total features in each group provided by the sum in difficulty was proposed. A systematic approach to the measurement of the difference in[13] for the selection of features from text documents was also proposed. As per the article, the new comparative methods give equivalent ranks to apply that have the same difference, but disregard the variance in their comparative text occurrences. With this in observance, the researchers proposed a generalized difference calculation methodology and compared it with 7 common FS methods with 7 datasets and a few classification methods, like Naive Bayes and Support Vector Machine .It can also be inferred that the collection of features is a critical open problem of the present period as per the discussion above. The researchers concentrated on this area and suggested various methods to challenge it from various views. There are several approaches, such as heuristic, statistical, synthetic, evolution-inspired, evolutionary computation and altitude-based, which are used to identify the major representation elements. The literature review of these methods indicates that there is no gift available and that every solution has its own advantages and disadvantages. For the set of features, the latest fuzzy rough range was used in $[14,15]$. The trouble with the current fuzzy rough system is that it will not preserve the ideal dependency feature that can eccentric the dataset. An improved form of fuzzy rough pack that fits into the dataset was introduced to overcome this problem. The Fuzzy neighborhood and the Parameterized Fuzzy relationship were used to specify the upper and lower bounds of the selected 
border. The Researchers [16] also addressed a system used to binarize unevenly illuminated texts using different fuzzy inclusion and entropy measuring techniques. They addressed the fact that the whole method is automatic and is an open technique which is frequently simplified in order to be successful in "difficult" photos with diverse realms and characteristics.

\section{METHODOLOGY}

The entire process mainly consists of three phases:

Phase-1: Different Texture data set has a large amount of texture images that allows you to load datasets. Preprocessing of the images (Reduction of Noise)

Phase-2: Feature Selection can be done by Fuzzy Inclusion and Entropy measures using existing and proposed methods.

Phase-3: Classification can be done by Fuzzy classifier and accuracy can be compared to different parameters.

\subsection{Datasets}

We have used three texture datasets such as Brodatz Texture Album, VisTex Dataset and DynTex Dataset for texture analysis and classification.

Brodatz Texture Dataset: The textures in Brodatz are quite well accepted and widely used in the classification of textures. 112 textures of the Brodatz texture dataset are collected[17].The Brodatz textures sample images are shown in Figure 1.

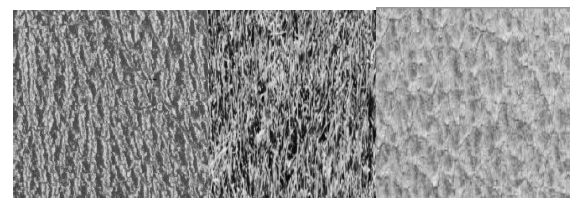

Figure 1: Brodatz texture sample images.

VisTex Dataset: VisTex (Vision Texture) is gathered from MIT (Massachusetts Institute of Technology) [18].This database is not frequently used, but it is more general. Figure 2 shows sample images from the dataset.

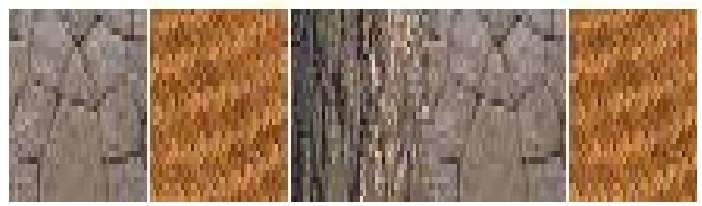

Figure 2: VisTex textures sample images.

DynTex Dataset: DynTex website is a vast set of interactive videos with good quality texture. Typically, dynamic textures are the result of processes like waves, smoke, and fire. Many realworld textures in the video database are dynamic and static. The sample images are shown in figure. 3 from the dataset.

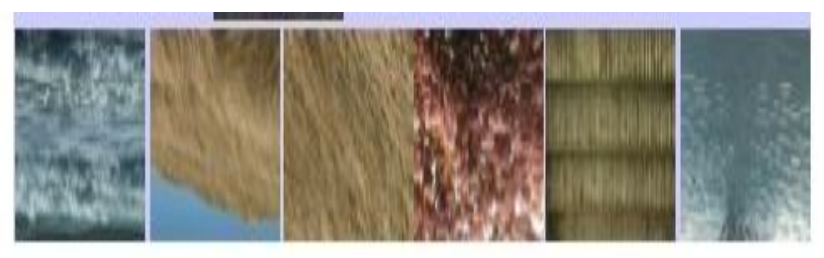

Figure s: vyll 1 ex textures sample inlages

\subsection{Preprocessing-Impact of Noise}

Different types of noises are introduced in the digital images at different stages of the preprocessing which degrades the images. Such deterioration has a major impact on the growth of many image processing techniques. Hence in many of techniques it is prerequisite to include a filter module before processing the digital image which is contaminated with the noise[19,20]. The challenge of many image processing technologies is the suppression of noise and the preservation of accurate edges and detailed image information.

\subsubsection{Impulse Noise}

Due to non-uniformness in the image noise corrupts the original pixels. This noise is caused due to sensors, hardware and transmission of data in noise channels. It is divided into two types like Specified impulse and Arbitrary impulse. Set pulse or stated pulse is generally known as salt and pepper noise. This noise appears in the picture as black and white specks. This noise is corrupting the values of higher or lower extreme intensity. Therefore, degradation is automatically applied to image causes for nonidentity of objects in the image.

\subsubsection{Gaussian Noise}

Generally, images are corrupted with different types of noises. Among them one of the noises is Gaussian noise which is an additive noise to an image. But due to power in the bandwidth Gaussian is added naturally such noise is called as additive white Gaussian noise. This noise is autonomous of the Gray Level Intensity value at every point. The main sources of occurring the Gaussiannoise is data acquisition, high temperature and transmission.

\subsubsection{Speckle Noise}

This noise is modeled with the value of random multiplication in relation to the pixel in the image 
that can be expressed in terms of $\mathrm{P}=\mathrm{I}+\mathrm{n} * \mathrm{I}$,If ' $\mathrm{P}$ ' shows the noise distribution in the image, ' $\mathrm{I}$ ' is the origin, and ' $n$ ' is the uniform noise in the picture with respect to Mean and Variance. Generally, the noise is observe dinremote sensing system due to the radiation in sensing the image using laser light and interaction of target area.

\subsection{Proposed Method}

Proposed Method is discussed in this section along with Global Method and Local Method.

\subsubsection{Global Thresholding using Fuzzy logic}

Suppose that the image is an $m \times n$ gray-scale format. We've got a fuzzy collection of m.n components by splitting the pixel intensities by 255 . Let the universal set of X. It's a white image of the same size, its counterpart is a completely black image, and $\mathrm{P}$ is a fully gray picture. Getting a group of inputs with various sizes and characteristics, the inclusion between set $\mathrm{A} \&$ set $\mathrm{X}$, set $\mathrm{A} \&$ set $\mathrm{P}$ and entropy e of $A$ is also calculated. We can inform you that

$\mathrm{S}_{1}(\mathrm{~A}, \mathrm{~B})=\Sigma \mathrm{x} \in \mathrm{Xmin}\left(\mathrm{m}_{\mathrm{A}}(\mathrm{x}), \mathrm{m}_{\mathrm{B}}(\mathrm{x})\right) / \Sigma \mathrm{x} \in \mathrm{Xm}_{\mathrm{A}}(\mathrm{x})=(\mathrm{A}$

$\cap B) / A$, if $A \neq \emptyset \&$

$\mathrm{S}_{1}(\mathrm{~A}, \mathrm{~B})=1$, if $\mathrm{A}=\emptyset$

$\mathrm{E}_{1}(\mathrm{~A})=\Sigma \mathrm{x} \in \mathrm{X} \quad \min \quad\left[\left(\mathrm{m}_{\mathrm{A}^{\mathrm{c}}}(\mathrm{x}), \mathrm{m}_{\mathrm{A}}(\mathrm{x})\right) / \Sigma \mathrm{x} \in \mathrm{X} \quad \max \right.$ $\left.\left(\mathrm{m}_{\mathrm{A}^{\mathrm{c}}}(\mathrm{x}), \mathrm{m}_{\mathrm{A}}(\mathrm{x})\right)\right]$.

$\mathrm{s}_{1}=\mathrm{S}_{1}(\mathrm{X}, \mathrm{A})$, where $\mathrm{X}$ is the Universal set of completely white image.

$s_{2}=S_{1}(A, \varnothing)$, where $X$ is the Universal set of completely black image.

$\mathrm{S}_{3}=\mathrm{S}_{1}(\mathrm{P}, \mathrm{A})$, where $\mathrm{P}$ iscompletelygrey image.

$\mathrm{s}_{4}=\mathrm{S}_{1}(\mathrm{~A}, \mathrm{P}), \mathrm{e}=\mathrm{E}_{1}(\mathrm{~A})$

The value ' $s 1$ ' indicates 'Brightness' of the image, the value 's2' indicates 'Darkness'and s3 \& s4 indiçâtes the greyness.

Global Method: Bogiatzis and Papadopoulos [21,22] is used a set of measure of fuzzy set defined as S1 but in the proposed process a fuzzy set S2 is measured completely equivalent to $\mathrm{S} 1$. Let us suppose that a pixel $\mathrm{p}$ and its $\mathrm{m} \times \mathrm{n}$ neighborhood $\mathrm{M}$. Then we test the fuzzy subset between the set $M$ and the set $X$, the preset and the $\mathrm{P}$ using $\mathrm{S} 1$. Now we have $\mathrm{S}_{1}=\mathrm{S}_{1}(\mathrm{X}, \mathrm{A}), \mathrm{S}_{2}=\mathrm{S}_{1}(\mathrm{~A}, \mathrm{~A}), \mathrm{S}_{3}=\mathrm{S}_{1}(\mathrm{P}, \mathrm{A})$, $\mathrm{S}_{4}=\mathrm{S}_{1}(\mathrm{~A}, \mathrm{P})$ and $\mathrm{e}=\mathrm{E}_{1}(\mathrm{~A})$.

The grouping of classes based on s 1 and s 2 is as follows:

Group 1:M with $\left[S_{1} \leq S_{2}\right]$ and $\left|S_{1}-S_{2}\right|>0.75$

Group 2: $M$ with $\left[S_{1} \leq S_{2}\right]$ and $0.5<\left|S_{1}-S_{2}\right| \leq 0.75$

Group 3: $M$ with $\left[S_{1} \leq S_{2}\right]$ and0.25 $<\left|S_{1}-S_{2}\right| \leq 0.5$

Group 4:M with $\left[S_{1} \leq S_{2}\right]$ and $\left|S_{1}-S_{2}\right| \leq 0.25$

Group 5: $M$ with $\left[S_{1}>S_{2}\right]$ and $\left|S_{1}-S_{2}\right|>0.75$

Group 6: M with $\left[S_{1}>S_{2}\right]$ and0.5 $<\left|S_{1}-S_{2}\right| \leq 0.75$
Group 7:M with $\left[\mathrm{S}_{1}>\mathrm{S}_{2}\right]$ and $0.25<\left|\mathrm{S}_{1}-\mathrm{S}_{2}\right| \leq 0.5$

Group 8:M with $\left[S_{1}>S_{2}\right]$ and $\left|S_{1}-S_{2}\right| \leq 0.25$

\section{Algorithm1: Global thresholding (General Method)}

1: Taking an image $\mathrm{i}_{\mathrm{m}}=\left\{\mathrm{pij} / \mathrm{p}_{\mathrm{ij}} \in \mathrm{M}\right.$, $0 \leq \mathrm{p}_{\mathrm{ij}} \leq 255, \mathrm{i}=1$ to $\mathrm{m}, \mathrm{j}=1$ to $\left.\mathrm{n}\right\}$

2: Build set $A$ of $m \cdot n$ elements with $\mathrm{mA}(\mathrm{x})=$ pij 255 for every pixel $\mathrm{x}$

3: Measure $\mathrm{S}_{1}, \mathrm{~S}_{2}, \mathrm{~S}_{3}, \mathrm{~S}_{4} \& \mathrm{e}$

4: Measure $r=r\left[S_{1}, S_{2}\right]$

5: Construct a fuzzy symmetrical

Triangular value

$\sim \mathrm{t}=[\mathrm{r}-\mathrm{c}, \mathrm{r}, \mathrm{r}+\mathrm{c}]$, where $\mathrm{c}=\left|\mathrm{S}_{3}-\mathrm{S}_{4}\right|$

6:Using ' $\mathrm{e}$ ' as the real value and evaluate

$\mathrm{t}_{1}=\mathrm{c}[\mathrm{e}-1]+\mathrm{r}$ and $\mathrm{t}_{2}=\mathrm{c}[1-\mathrm{e}]+\mathrm{r}$

7: Set $\mathrm{t}=\mathrm{t}_{1}$ or $\mathrm{t}_{2}$

8: Binary image with threshold ' $\mathrm{t}$ '

\subsubsection{Local Thresholding using Fuzzy logic}

Algorithm 2: Local Thresholding

1: Let's consider $\mathrm{m} \times \mathrm{n}$ neighborhood of pixel $\mathrm{p}$ \&fuzzy set $M$ of m.n of pixelElements by dividing the gray intensity by 255 .

2: Measure $S_{1}, S_{2}, S_{3}, S_{4} \&$ e

3: To define set $\mathrm{M}$ and compute $\mathrm{r}$ on the basis of its group

4: Construct a fuzzy symmetrical Triangular Value

$\sim \mathrm{t}=[\mathrm{r}-\mathrm{c}, \mathrm{r}, \mathrm{r}+\mathrm{c}]$, where $\mathrm{c}=\left|\mathrm{S}_{3}-\mathrm{S}_{4}\right|$

5: Using ' $\mathrm{e}$ ' as the real value and evaluate

$\mathrm{t}_{1}=\mathrm{c}[\mathrm{e}-1]+\mathrm{r}$ and $\mathrm{t}_{2}=\mathrm{c}[1-\mathrm{e}]+\mathrm{r}$

6: Set $\mathrm{t}=\mathrm{t}_{1}$ or $\mathrm{t}_{2}$

7: Binary pixel $\mathrm{p}$ with threshold ' $\mathrm{t}$ '

\section{a) Implementation of algorithm2: Local Method}

The implementation is based on some standard $r$ for each of eight classes.

Set $r=0.49[0.49-0.495]$, WhenMis in classI Set $r=0.48[0.48-0.49]$, When $M$ is in class II Set $r=0.47[0.47-0.48]$, When $M$ is in class III Set $r=0.46[0.46-0.47]$, When $M$ is in class IV Set $r=0.43[0.43-0.44]$, When $\mathrm{M}$ is in class $\mathrm{V}$ Set $r=0.44(0.44-0.45]$, When $\mathrm{M}$ is in class VI Set $r=0.45[0.45-0.46]$, When $M$ is in class VII Set $r=0.46$ [0.46-0.47], WhenM is in class VIII Also set the values as per step 6 .

If $\mathrm{S}_{1} \leq \mathrm{S}_{2}$, then $\mathrm{t}=\mathrm{t}_{1}$ else $\mathrm{t}=\mathrm{t}_{2}$ If $\mathrm{t} \leq 0$ then $\mathrm{t}=0.01$ and If $\mathrm{t} \geq 1$ then $\mathrm{t}=0.99$ 


\section{b) Implementation of algorithm 2: Proposed Method}

Implementation of the proposed algorithm with values of $\mathrm{r}$ not specified, but isdetermined on the basis of values of $S_{1}$ and $S_{2}$.

Set $r=0.41+0.08 S_{2}$, When $M$ is in class I

Set $r=0.39+0.112 S_{2}$, When M is in class II

Set $r=0.37+0.153 S_{2}$, When $M$ is in class III

Set $r=0.35+0.206 S_{2}$, When $M$ is in class IV

Set $r=0.41+0.036 S_{1}$, When $M$ is in class $V$

Set $r=0.39+0.098 S_{1}$, When $M$ is in class VI

Set $r=0.37+0.115 S_{1}$, When $M$ is in class VII

Set $r=0.35+0.206 S_{1}$, When $M$ is in class VIII

\subsection{Matching Parameters}

After successful completion of detection matching is applied by using certain parameters to estimate accuracy.

Jaccard: Jaccard coefficient $=\frac{a}{a+b+c}$

Braun: Braun coefficient $=\frac{a}{\max (a+b, b+c)}$

Ochiai: Ochiai coefficient $=\frac{a}{\operatorname{sqrt}((a+b)(a+c))}$

Rogers: Rogers coefficient $=\frac{a+d}{a+d+2(b+c)}$

Simpson:
Simpson coefficient $=\frac{a}{\min (a+b, a+c)}$

Where ' $a$ ' is the total number of features shown in B but not in $\mathrm{A}$ and ' $\mathrm{b}$ ' is the total number of features shown in A but not in B.

\section{RESULTS AND DISCUSSIONS}

A systematic performance evaluation is done to determine the efficiency of the texture classification of the proposed process using the current Global Process and the Local Method using different parametric steps. In the experimental setup, the input images were categorized using three different forms of noise, namely Gaussian, Salt-Pepper and Speckle Noise, and the effectiveness of the proposed procedure is clarified. The matching parameters Jaccard, Bruan, Ochiai, Simpson and Rogers are evaluated by inducing a range of noise percentages from $0 \%$ to $25 \%$ on the input images. The proposed method is evaluated in 3 different modes, Global method, Local method and Proposed method. In each method, the performance values are evaluated using different noise percentages from 0 to 25 with a difference of $5 \%$ at each moment.

Table 4: Performance comparison of the proposed approach with the local and global approaches in the presence of Salt-Pepper Noise.

\begin{tabular}{|c|c|c|c|c|c|c|c|}
\hline Method & $\begin{array}{l}\text { Noise } \\
\text { Type }\end{array}$ & $\begin{array}{c}\text { Noise } \\
\text { Percentage }\end{array}$ & JACCARD & BRAUN & OCHIAI & SIMPSON & ROGERS \\
\hline \multirow{6}{*}{$\begin{array}{l}\text { Global } \\
\text { Method }\end{array}$} & \multirow{18}{*}{ 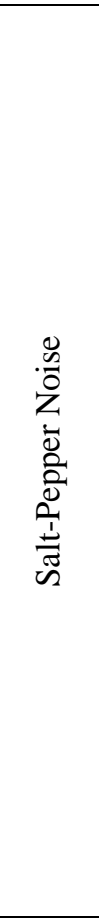 } & 0 & 0.932 & 0.959 & 0.9648 & 0.9707 & 0.9151 \\
\hline & & 5 & 0.8759 & 0.9211 & 0.93 & 0.947 & 0.845 \\
\hline & & 10 & 0.8258 & 0.8849 & 0.9 & 0.9253 & 0.7822 \\
\hline & & 15 & 0.7866 & 0.8464 & 0.88 & 0.896 & 0.7336 \\
\hline & & 20 & 0.75 & 0.8 & 0.83 & 0.85 & 0.6875 \\
\hline & & 25 & 0.72 & 0.7794 & 0.78 & 0.82 & 0.6503 \\
\hline \multirow{6}{*}{$\begin{array}{l}\text { Local } \\
\text { Method }\end{array}$} & & 0 & 0.942 & 0.9655 & 0.97 & 0.973 & 0.9282 \\
\hline & & 5 & 0.8885 & 0.9319 & 0.935 & 0.9486 & 0.8623 \\
\hline & & 10 & 0.8385 & 0.8941 & 0.91 & 0.9259 & 0.801 \\
\hline & & 15 & 0.79 & 0.8577 & 0.883 & 0.8985 & 0.7434 \\
\hline & & 20 & 0.751 & 0.81 & 0.838 & 0.859 & 0.6957 \\
\hline & & 25 & 0.728 & 0.79 & 0.79 & 0.83 & 0.6585 \\
\hline \multirow{6}{*}{$\begin{array}{c}\text { Proposed } \\
\text { Method }\end{array}$} & & 0 & 0.9468 & 0.9704 & 0.9721 & 0.975 & 0.9346 \\
\hline & & 5 & 0.900 & 0.9373 & 0.943 & 0.9501 & 0.8689 \\
\hline & & 10 & 0.850 & 0.904 & 0.9153 & 0.937 & 0.8084 \\
\hline & & 15 & 0.820 & 0.8704 & 0.885 & 0.9047 & 0.7479 \\
\hline & & 20 & 0.757 & 0.8221 & 0.839 & 0.89 & 0.7038 \\
\hline & & 25 & 0.7350 & 0.7997 & 0.81 & 0.8746 & 0.6898 \\
\hline
\end{tabular}


M.Subba Rao et al., International Journal of Advanced Trends in Computer Science and Engineering, 9(4), July - August 2020,4907 - 4917

Table 5: Quality comparison of the proposed system with the local and global approaches in the presence of Gaussian Noise.

\begin{tabular}{|c|c|c|c|c|c|c|c|}
\hline Method & $\begin{array}{l}\text { Noise } \\
\text { Type }\end{array}$ & $\begin{array}{c}\text { Noise } \\
\text { Percentage }\end{array}$ & JACCARD & BRAUN & OCHIAI & SIMPSON & ROGERS \\
\hline \multirow{6}{*}{$\begin{array}{l}\text { Global } \\
\text { Method }\end{array}$} & \multirow{18}{*}{ 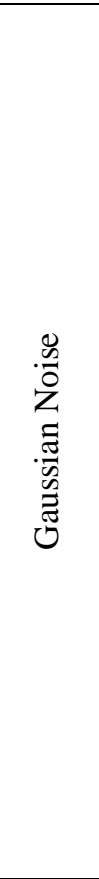 } & 0 & 0.8846 & 0.8982 & 0.9397 & 0.9832 & 0.8642 \\
\hline & & 5 & 0.7398 & 0.7434 & 0.8594 & 0.9905 & 0.6453 \\
\hline & & 10 & 0.639 & 0.6416 & 0.7984 & 0.9936 & 0.5165 \\
\hline & & 15 & 0.6065 & 0.6091 & 0.7798 & 0.9983 & 0.4558 \\
\hline & & 20 & 0.5825 & 0.5826 & 0.7632 & 0.9987 & 0.3859 \\
\hline & & 25 & 0.5162 & 0.55 & 0.75 & 0.9989 & 0.3112 \\
\hline \multirow{6}{*}{$\begin{array}{l}\text { Local } \\
\text { Method }\end{array}$} & & 0 & 0.9054 & 0.915 & 0.95 & 0.987 & 0.8785 \\
\hline & & 5 & 0.7758 & 0.7816 & 0.87 & 0.9934 & 0.7203 \\
\hline & & 10 & 0.6764 & 0.6772 & 0.822 & 0.9943 & 0.5448 \\
\hline & & 15 & 0.6262 & 0.6262 & 0.79 & 0.9987 & 0.4655 \\
\hline & & 20 & 0.6074 & 0.62 & 0.785 & 0.9989 & 0.4216 \\
\hline & & 25 & 0.5855 & 0.6 & 0.766 & 0.9997 & 0.3987 \\
\hline \multirow{6}{*}{$\begin{array}{l}\text { Proposed } \\
\text { Method }\end{array}$} & & 0 & 0.9273 & 0.9388 & 0.9626 & 0.9885 & 0.9089 \\
\hline & & 5 & 0.7944 & 0.7955 & 0.8911 & 0.9983 & 0.7215 \\
\hline & & 10 & 0.6851 & 0.6852 & 0.83 & 0.9953 & 0.5531 \\
\hline & & 15 & 0.6362 & 0.6363 & 0.7975 & 0.9989 & 0.4785 \\
\hline & & 20 & 0.629 & 0.63 & 0.7987 & 0.9992 & 0.4664 \\
\hline & & 25 & 0.6 & 0.62 & 0.792 & 0.9999 & 0.4587 \\
\hline
\end{tabular}

Table 6: Performance comparison of the proposed approach with the local and global methods in the presence of Speckle Noise.

\begin{tabular}{|c|c|c|c|c|c|c|c|}
\hline Method & $\begin{array}{l}\text { Noise } \\
\text { Type }\end{array}$ & $\begin{array}{c}\text { Noise } \\
\text { Percentage }\end{array}$ & JACCARD & BRAUN & OCHIAI & SIMPSON & ROGERS \\
\hline \multirow{6}{*}{$\begin{array}{l}\text { Global } \\
\text { Method }\end{array}$} & \multirow{18}{*}{ 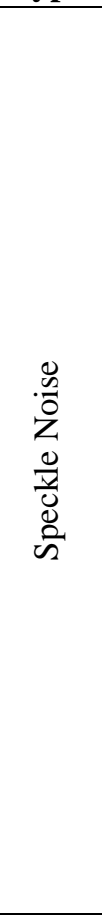 } & 0 & 0.9278 & 0.9594 & 0.9397 & 0.9658 & 0.9181 \\
\hline & & 5 & 0.8892 & 0.9365 & 0.8799 & 0.9463 & 0.8757 \\
\hline & & 10 & 0.8437 & 0.9143 & 0.7984 & 0.9386 & 0.8473 \\
\hline & & 15 & 0.7936 & 0.8942 & 0.7798 & 0.9324 & 0.8234 \\
\hline & & 20 & 0.7568 & 0.8795 & 0.7632 & 0.9287 & 0.807 \\
\hline & & 25 & 0.7192 & 0.8512 & 0.75 & 0.9158 & 0.8 \\
\hline \multirow{6}{*}{$\begin{array}{l}\text { Local } \\
\text { Method }\end{array}$} & & 0 & 0.9468 & 0.9765 & 0.9626 & 0.99 & 0.9611 \\
\hline & & 5 & 0.8931 & 0.9565 & 0.9414 & 0.9857 & 0.9311 \\
\hline & & 10 & 0.8627 & 0.9375 & 0.9264 & 0.9863 & 0.9116 \\
\hline & & 15 & 0.8398 & 0.9166 & 0.9131 & 0.9867 & 0.8869 \\
\hline & & 20 & 0.8239 & 0.8991 & 0.9038 & 0.9881 & 0.8674 \\
\hline & & 25 & 0.81 & 0.8732 & 0.8954 & 0.9896 & 0.84 \\
\hline \multirow{6}{*}{$\begin{array}{l}\text { Proposed } \\
\text { Method }\end{array}$} & & 0 & 0.9686 & 0.9811 & 0.9855 & 0.9987 & 0.965 \\
\hline & & 5 & 0.947 & 0.9655 & 0.9754 & 0.9934 & 0.9416 \\
\hline & & 10 & 0.927 & 0.9436 & 0.9634 & 0.9983 & 0.9149 \\
\hline & & 15 & 0.9055 & 0.9292 & 0.9555 & 0.9986 & 0.8977 \\
\hline & & 20 & 0.888 & 0.9069 & 0.9429 & 0.9996 & 0.8714 \\
\hline & & 25 & 0.862 & 0.8923 & 0.9355 & 0.9999 & 0.865 \\
\hline
\end{tabular}

The performance of proposed approach with local and global methods in the presence of various noises such as Salt-Pepper, Gaussian and Speckle noises are shown in tables 4,5 and 6 respectively. Table 4 demonstrates the efficiency of the proposed method with the Local and Global methods in the presence of Salt-Pepper Noise with specific corresponding 
parameters.For each form, the noise percentages vary with a 5 percent difference varying from 0 to 25 , and the values are described in Table 4. Table 5 shows the performance of the proposed method with the Local and Global methods in the presence of Gaussian Noise with different matching parameters. In each form, the noise percentages vary with a 5 percentdifference between 0 and 25 , and the values are described in Table 5.Table 6 demonstrates the efficiency of the Proposes method using the Local method and the Global method in the presence of Speckle Noise with specific matching parameters.
For each system, the noise percentages vary with a 5 percent difference between 0 and 25 , and the values are described in Table 6.

Figure 4-8 demonstrates the classification accuracy of Jaccard, Braun, Ochiai, Simpson and Rogers on proposed system with specific noise percentages using local and global methods for Salt-Pepper, Gaussian and Speckle noises. on proposed method with local and global methods for Salt-Pepper, Gaussian and Speckle noises with various noise percentages.

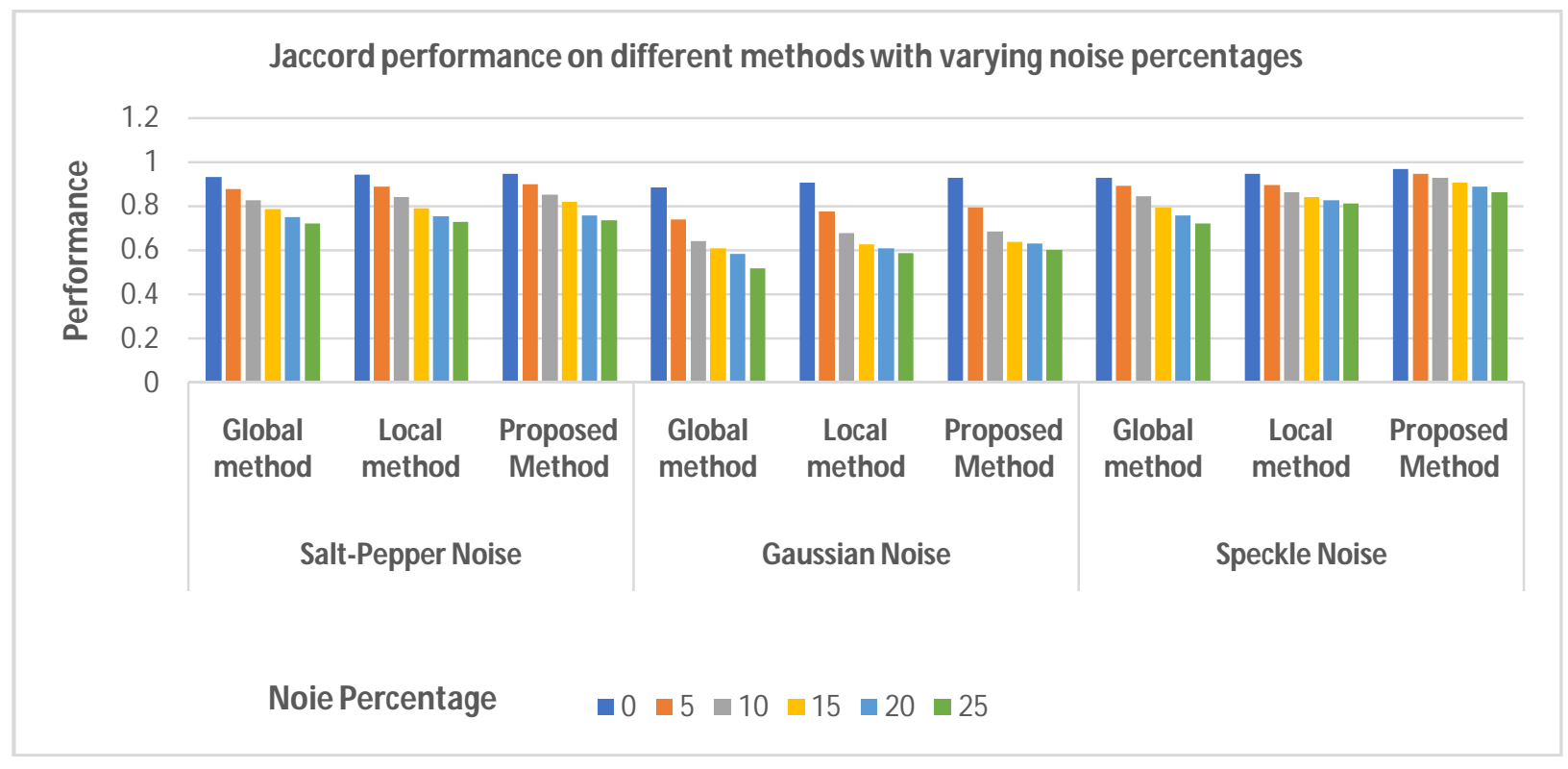

Figure 4: Accuracy comparison of the Jaccard classification test for the proposed method with the Global method and the Local method for Salt-Pepper, Gaussian Noise and Speckle Noise.

Figure 4 demonstrates the efficiency of the Jaccard classification of the proposed system with the Local method and the Global method in the presence of Salt-Pepper, Gaussian noise and Speckled noise. From Figure 4, the efficiency of the Jaccard calculation is decreasing with regard to the rise in the percentage of noise. Figure 4 shows that the proposed method presented the best classification results with respect to the global method and the local method in the presence of different noises with variable percentages. In Figure 4, the proposed approach has achieved the highest performance with respect to Speckle Noise and has reported low performance with respect to Gaussian Noise. Figure 5 demonstrates the efficiency of the Braun classification test of the proposed system with the Local method and the Global method in the presence of Salt-Pepper, Gaussian noise and Speckled noise. Figure 5 indicates a decline in the efficiency of the Braun calculation with regard to the rise in the amount of noise. Figure 5 shows that the proposed method presented the best classification results with respect to the Global method and the Local method in the presence of different noises with variable percentages. Figure 5 indicates that the proposed approach has achieved the best efficiency with various amounts of noise with respect to the Speckle noise and has recorded low performance with respect to Gaussian noise. 


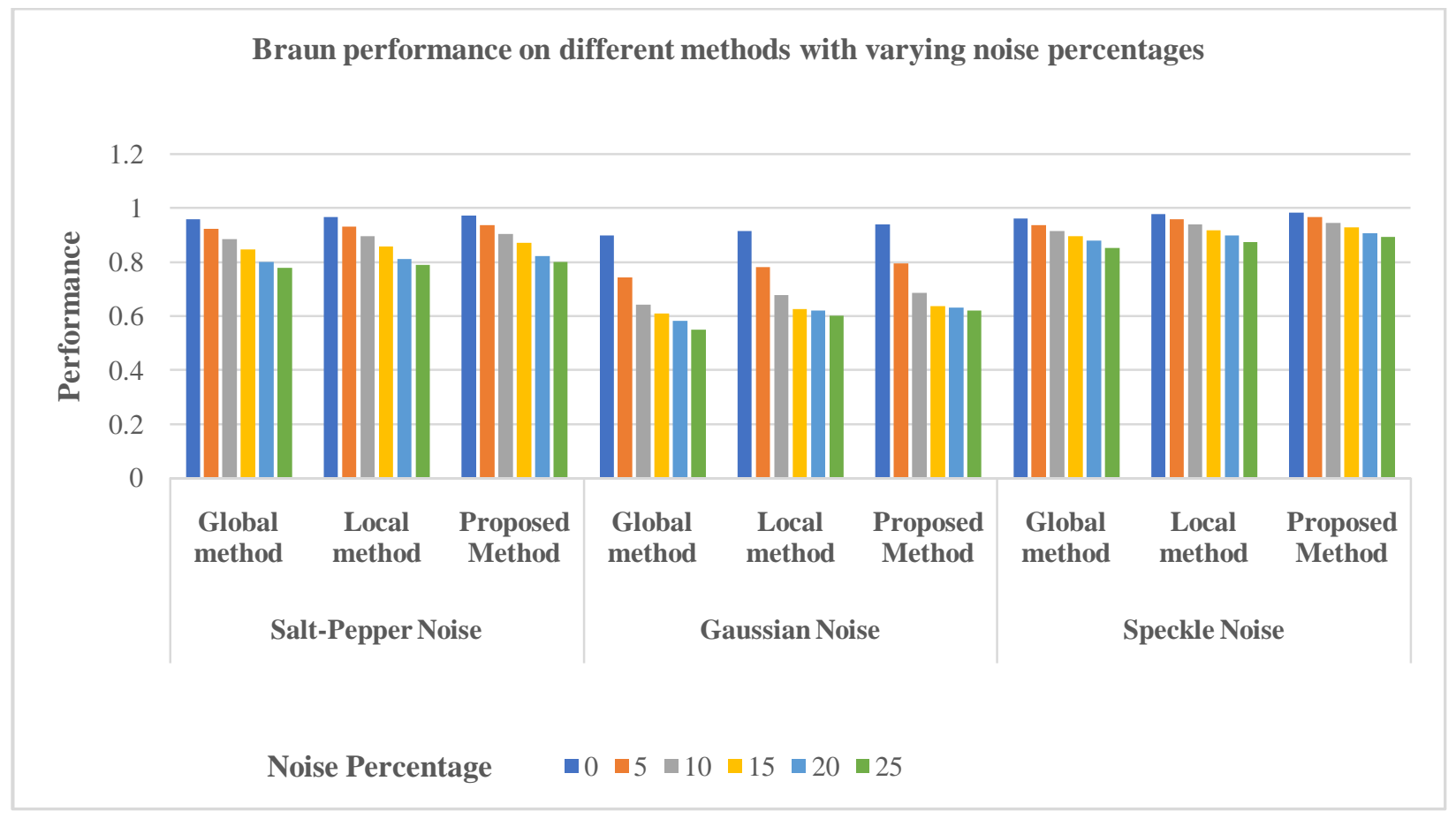

Figure 5: Performance comparison of Braun classification measure on proposed method with Global method and Local method with respect to Salt-Pepper noise, Gaussian Noise and Speckle noise.

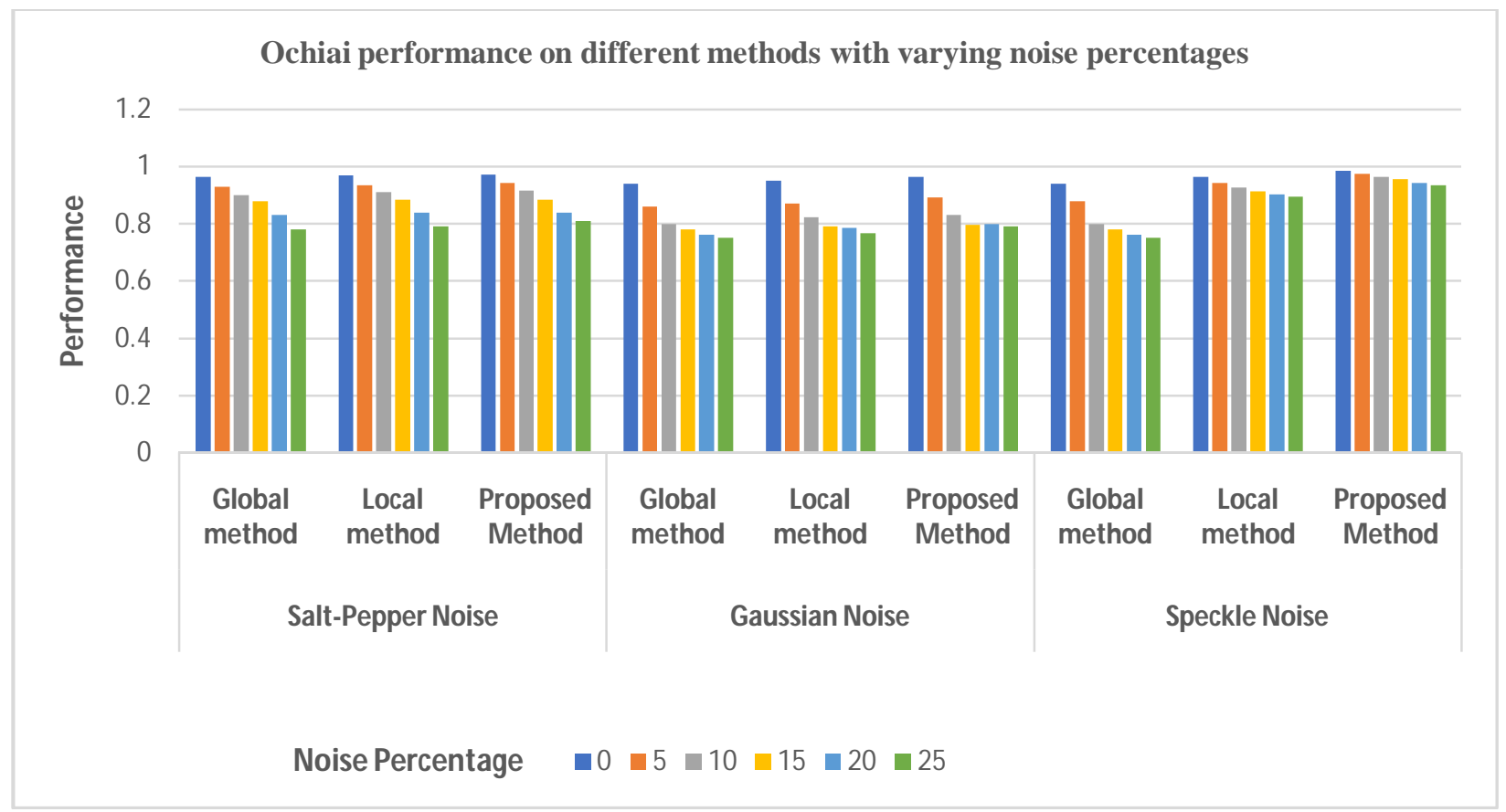

Figure 6: Efficiency comparison of the Ochiai classification measure on the proposed system with the Global method and the Local method for Salt-Pepper, Gaussian Noise and Speckle Noise. 


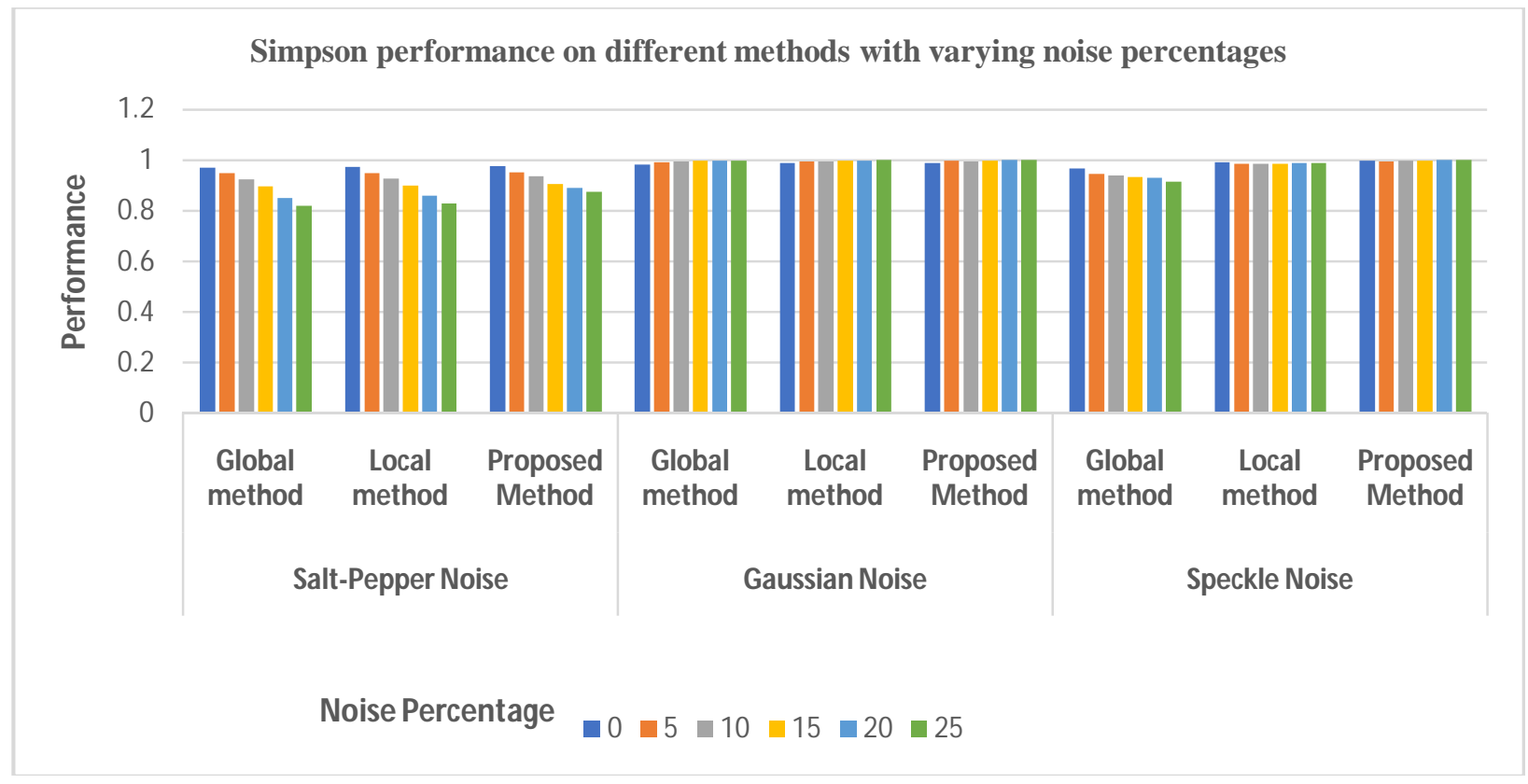

Figure 7: Performance comparison of Simpson classification measure on proposed method with Global method and Local method with respect to Salt-Pepper noise, Gaussian Noise and Speckle noise.

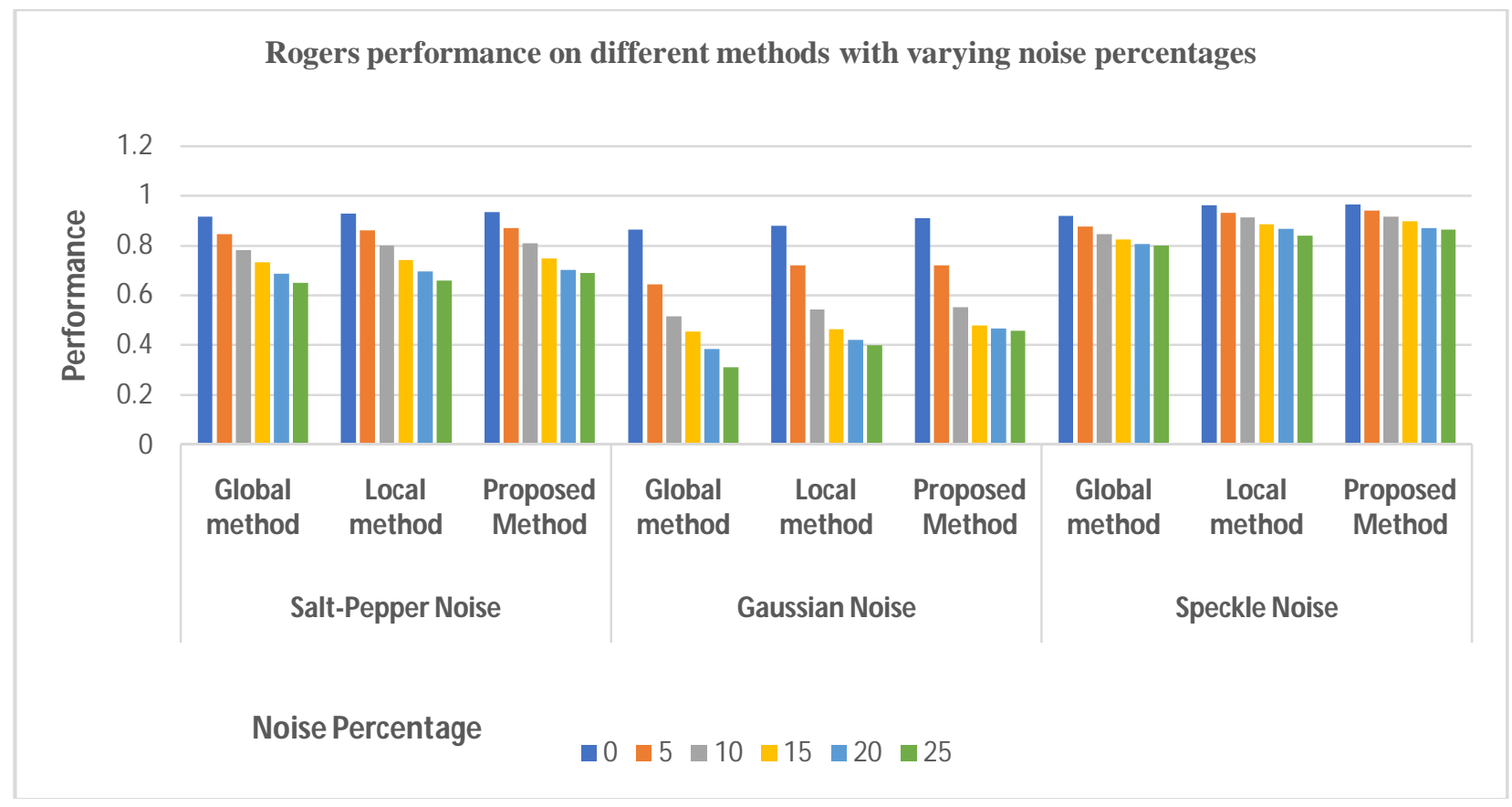

Figure 8: Performance comparison of Rogers classification measure on proposed method with Global method and Local method with respect to Salt-Pepper noise, Gaussian Noise and Speckle noise 
Figure 6 shows the efficiency of the Ochiai classification measure of the proposed method with the Local method and the Global method in the presence of Salt-Pepper, Gaussian noise and Speckled noise. Figure 6 indicates a decline in the efficiency of the Ochiai calculation with respect to the rise in the amount of noise. Figure 6 shows that the proposed method presented the best classification results with respect to the global method and the local method in the presence of different noises with variable percentages. The suggested approach has obtained the best efficiency with variable noise percentages in the presence of Speckle Noise in Figure 6 and has recorded a low performance with respect to Gaussian noise.

Figure 7 demonstrates the efficiency of the Simpson classification test of the proposed local method and the global method in the presence of Salt-Pepper, Gaussian noise and Speckled noise. From Figure 7, when a variable Salt-Pepper noise is introduced into the input image, the output of the Simpson metric decreases with respect to the change in the percentage of the noise and is inconsistent with Gaussian noise and Speckle noise. The efficiency of the Simpson metric is growing with respect to the percentage of noise in the presence of Gaussian and Speckle noise. Figure 7 shows that the efficiency of the proposed method is that with respect to the increase in noise and has achieved the best classification accuracy with respect to the Global method and the Local method in the presence of different noises with variable percentages. In Figure 7 , the proposed approach has improved performance with variable noise percentages in the presence of Speckle Noise and has reported poor output with respect to Salt-Pepper Noise.

Figure 8 demonstrates the efficiency of the Rogers classification test of the proposed system with the Local method and the Global method in the presence of Salt-Pepper, Gaussian noise and Speckled noise. Figure 8 indicates a decline in the efficiency of the Rogers metric with respect to the rise in the amount of noise. Figure 8 shows that the proposed method presented the best classification results with respect to the global method and the local method in the presence of different noises with variable percentages. Figure 8 indicates that the proposed approach has achieved the best efficiency with various amounts of noise with respect to the Speckle noise and has recorded low performance with respect to Gaussian noise.

\section{CONCLUSION}

Local and global threshold techniques focused on diffuse inclusion and calculation of entropy are discussed for the classification of textures for images with irregular lighting. The efficiency of the proposed technique has also been studied under the influence of noise in the images. Three different noises in different proportions were applied to the original image and the statistical measurements of Jaccard, Bruan, Ochiai, Simpson and Rogers were analyzed in order to understand the efficiency of the proposed algorithm and were also correlated with current local and global methods. The proposed approach achieved better performance in the presence of Speckle noise and poor performance in the case of Gaussian noise with variable noise percentages ranging from 0 to 25 percent. It was concluded from the experimental study that the proposed approach effectively classifies a multi-resolution image and works well for low contrast and overlapping images.

\section{REFERENCES}

1. M.Tuceryan, and A.K. Jain, "Texture Analysis," The Handbook of Pattern Recognition and Computer Vision (2nd Ed), World Scientific Publishing, Singapore, pp. 207-248, 1998.

https://doi.org/10.1142/9789812384737_0007

2. B. S. Manjunathi and W. Y. Ma, "Texture features for browsing and retrieval of image data," IEEE Trans. Pattern Anal. Mach. Intell., vol. 18, no. 8, pp. 837-842, Aug. 1996

3.Chengjun Liu, Wechsler, H., "A shape- and texture-based enhanced Fisher classifier for face recognition," IEEE Transactions on Image Processing, vol.10, no.4, pp.598-608, Apr 2001

4.Davis Larry"Image Texture Analysis Techniques:A Survey",Springerconference on Digital ImagProcessing, 1981.

5. Ramírez-Gallego, S., Krawczyk, B., García, S., Woźniak, M., \& Herrera, F. (2017). "A survey on data pre-processing for data stream mining: current status and future directions". Neurocomputing, 239, 39-57. https://doi.org/10.1016/j.neucom.2017.01.078 6.Noh, S., Zoltowski, M. D., Sung, Y., \& Love, D. J. (2014). "Pilot beam pattern design for channel estimation in massive MIMO systems". IEEE Journal of Selected Topics in Signal Processing, 8(5), 787-801. 
6.Melin, P., \& Castillo, O. (2014). "A review on type-2 fuzzy logic applications in clustering, classification and pattern recognition". Applied soft computing, 21, 568-577.

7.Akhtar, M. S., Gupta, D., Ekbal, A.\& Bhattacharyya, P, (2017). "Featureselection and ensemble construction: A two-step method foraspect-basedSentimentanalysis". Knowledge-

Based Systems, 125, 116-135

8 M.Subba Rao, Dr. B. Eswara Reddy "Comparative Analysis of pattern RecognitionMethods: An Overview" IJCSE, 2011 Volume 2, Issue 3, ISSN: 0976-516.

9.Fong, S., Wong, R., \&Vasilakos, A. (2016). "Accelerated PSO swarm search feature selection for data stream mining big data". IEEE transactions on services computing, (1), 1-1.

10.Uysal, A. K. (2017). "An improved global feature selection scheme for text classification". Expert systems with Applications, 43, 82-92.

11.Agnihotri, D., Verma, K., \& Tripathi, P. (2017).

"Variable Global Feature Selection heme for automatic classification of text documents". Expert Systems with Applications, 81, 268281.

https://doi.org/10.1016/j.eswa.2017.03.057

12. Rehman, A., Javed, K., \&Babri, H. A. (2017).

"Feature selection based on a normalized difference measure for text classification'Information Processing \& Management, 53(2), 473-489.

13.Wang, C., Qi, Y., Shao, M., Hu, Q., Chen, D., Qian, Y., \& Lin, Y. (2017). "A fitting model for feature selection with fuzzy rough sets". IEEE Transactions on Fuzzy Systems, 25(4), 741-753.

14.Hussain. S, "A Methodology to Predict the Instable Classes", 32nd ACM Symposium Bon Applied Computing (SAC) Morocco, 4th to 6th April 2017

15.Athanasios C. Bogiatzis, and Basil K.Papadopoulos, "Binarization of texts with varying lighting conditions using fuzzy inclusion and entropy measures", AIP Conference Proceedings 1978, 290006, 2018. https://doi.org/10.1063/1.5043913

16.Brodatz Textures: A Photographic Album for Artists and Designers, Dover, New York, 1996.

17.Jing Yi Tou1, Yong Haur Tay1, Phooi Yee Lau "Recent Trends In Texture Classification: A Review", Symposium on Progress in Information \& Communication Technology 2009.

18.M. TulinYıldırım, Alper Bas, turk and M.EminYuksel, "Impulse Noise Removal from Digital Images by a Detail-Preserving Filter Based on Type-2 Fuzzy Logic", IEEE transactions on fuzzy systems, pp-920-928,2008.

19.Tom Mélange, Mike Nachtegael, and Etienne E. Kerre, "Fuzzy Random Impulse Noise Removal from Color Image Sequences", IEEE transactions on image processing, pp-959-970, 2011.

https://doi.org/10.1109/TIP.2010.2077305

20.Bogiatzis A, Papadopoulos B, "Binarization of texts with varying lighting conditions using fuzzy inclusion and entropy measures", Int Conf Num Anal Appl Math 1978(1), 290006, 2018.

21.Bogiatzis A, Papadopoulos B, "Producing fuzzy inclusion and entropy measures and their application on global image thresholding", Evolving Systems.

22.B. Kishore, V.Vijay Kumar, “ Gradient ternary Transition based Cross Diagonal Texture Matrix for Texture Classification", IJATCSE, Vol 8, No.4, July-August 2019

https://doi.org/10.30534/ijatcse/2019/47842019

23.AbeerShamaileh "Different binary Local

Operators for Texture Classification-A Comparative Study", IJATCSE, Vol 8, No.3, MayJune 2019

https://doi.org/10.30534/ijatcse/2019/85832019

24.K.S.R.K.Sarma, “ Local Gradient Dual Coding Book Framework for effective Texture Classification" , IJATCSE, Vol. 8, No. 4, JulyAugust 2019. 